Pacific Journal of Mathematic 


\title{
A DECOMPOSITION OF ADDITIVE SET FUNCTIONS
}

\author{
WAYNE C. BeLL
}

In this paper it is demonstrated that if $\mu$ is an additive function from a field $F$ into the nonnegative reals, then $\mu$ can be separated into two mutually singular parts, $\mu_{1}$ and $\mu_{2}$, where $\mu_{1}$ is representable in the sense that its Lebesgue decomposition projection operator has a refinement integral representation and $\mu_{2}$ is such that for each $E \in F$ the contraction of $\mu_{2}$ to $E$ is representable iff $\mu_{2}(E)=0$. If $\mu_{2}$ is maximal, then the decomposition is unique.

1. Introduction. Suppose $S$ is a set, $F$ a field of subsets of $S, b(F)$ the set of bounded functions from $F$ into $R$, and $b a(F)$ the set of functions in $b(F)$ which are additive on disjoint elements of $F$. For $H \subseteq b a(F)$ denote by $H^{+}$the set of nonnegative valued elements of $H$ and let $\mu$ be in $b a(F)^{+}$. For $\lambda \in b a(F)^{+}$denote by $A_{\lambda}$ the set of elements in $b a(F)$ which are absolutely continuous with respect to $\lambda$ and by $\alpha_{\lambda}$ the Lebesgue decomposition projection operator for $\lambda$, i.e., for $\eta \in b a(F), \alpha_{\lambda}(\eta)$ is that part of $\eta$ which is absolutely continuous with respect to $\lambda[5]$. For $\lambda \in b a(F)^{+}$. we say that $\lambda$ is representable if there exists a $g: F \rightarrow \boldsymbol{R}$ such that $\alpha_{\lambda}(\eta)=\int g \eta$ for each $\eta \in b a(F)$ in which case $g$ will be said to represent $\lambda$.

2. Preliminary theorems. All integrals in this paper are refinement limits of sums over finite subdivision of $S$ by elements of $F$. If $\beta: F \rightarrow \boldsymbol{R}$ and $\int_{S} \beta(I)$ exists we will denote by $\int \beta$ the function $\left\{\left(v, \int_{v} \beta(I)\right) \mid v \in F\right\}$. For further details concerning the integral and 2. K. 1 and 2. K. 2 below see [1].

TheOREM 2. K. 1. If $\alpha: F \rightarrow \boldsymbol{R}$ and $\int_{S} \alpha(I)$ exists, then

$$
\int_{S}\left|\alpha(v)-\int_{v} \alpha(I)\right|
$$

exists and is zero. Consequently, if $\beta \in b(F)$ and $v \in F$, then $\int_{v} \beta(I) \int_{I} \alpha(J)$ exists iff $\int_{v} \beta(I) \alpha(I)$ exists in which case they are equal.

Proof. [9].

Corollary 2. K. 2. If $\alpha: F \rightarrow \boldsymbol{R}$ and $\beta: F \rightarrow \boldsymbol{R}$ and each of 
$\int_{S} \alpha(I)$ and $\int_{S} \beta(I)$ exists and $M$ is either max or min then $\int M\{\alpha, \beta\}$
exists iff $\int M\left\{\int \alpha, \int \beta\right\}$ exists in which case they are equal.

Proof. [1].

Notice that if $h$ represents $\mu$, then for $\lambda \in b a(F)^{+}$we have $0 \leqq \alpha_{\mu}(\lambda)=\int h \lambda \leqq \lambda$ so that $\int h \lambda=\int \max \{0, \min \{h, 1\}\} \lambda$ and therefore $h$ can be replaced by a bounded function. Also any representation for $\mu$ which is valid for $b a(F)^{+}$is valid for $\eta \in b a(F)$ since $\alpha_{\mu}(\eta)=\alpha_{\mu}\left(\eta^{+}\right)-\alpha_{\mu}\left(\eta^{-}\right)$[5] where $\eta^{+}$and $\eta^{-}$are the positive and negative parts of $\eta$, respectively. Consequently we will restrict our attention to $b a(F)^{+}$.

We will also have need of the following theorem due to Appling.

TheOREM 2.A. If $\mu \in b a(F)^{+}, \eta \in A_{\mu}, \beta \in b(F)$ and $\int \beta \mu$ exists, then $\int \beta \eta$ exists.

\section{Proof. [3].}

If in subsequent statements the existence of a given integral or its equivalence to a given integral is an immediate consequence of the statements of this section, the integral will often only be written and the proof of existence or equivalence will be left to the reader.

3. Two lemmas. By the remarks of the previous section if $\mu$ has a representing function, then it has a bounded representing function which, by the following lemma we may assume to be the characteristic function of some subset of $F$.

Lemma 3.1. Suppose $h \in b(F)$ and for each $\lambda \in b a(F)^{+}$we have $\int h \lambda$ exists and is equal to $\int h \int h \lambda$. Then there exists a $g: F \rightarrow\{0,1\}$ such that for each $\lambda \in b a\left(F^{\prime}\right)^{+}$we have $\int g \lambda$ exists and is equal to $\int h \lambda$.

Proof. Let $\alpha=h^{2}, \beta=\min \{\alpha, 1\}$ and suppose $\lambda \in b a(F)^{+}$. It is an easy consequence of 2.K.1 and 2.K.2 that

$$
\int \alpha \lambda=\int \alpha^{2} \lambda=\int \alpha \int \alpha \lambda=\int h \lambda \text { and } \int \beta \lambda=\int \beta \int \beta \lambda=\int \beta^{2} \lambda .
$$




$$
\int \alpha \lambda \leqq \int \max \{\alpha, 1\} \lambda-\lambda+\lambda \leqq \int \max \{\alpha, 1\}(\max \{\alpha, 1\}-1) \lambda+\lambda=\lambda
$$

hence $\int \beta \lambda=\int \min \{\alpha, 1\} \lambda=\int \alpha \lambda=\int h \lambda$. Now

$$
\begin{aligned}
0 & \leqq \int \min \{\beta, 1-\beta\} \lambda=\int(1-\beta) \min \{\beta, 1-\beta\} \lambda+\int \beta \min \{\beta, 1-\beta\} \lambda \\
& =\int \min \left\{\beta-\beta^{2},(1-\beta)^{2}\right\} \lambda+\int \min \left\{\int \beta^{2} \lambda, \int \beta \lambda-\int \beta^{2} \lambda\right\} \\
& =\int \min \left\{\int \beta \lambda-\int \beta^{2} \lambda, \int(1-\beta)^{2} \lambda\right\}+0=0 .
\end{aligned}
$$

For each $v \in F \quad$ let $l(v)=\left\{\begin{array}{ll}\beta(v) & \text { if } \beta(v) \leqq 1 / 2 \\ 0 & \text { otherwise. }\end{array}\right.$ Then $0 \leqq l \leqq$ $\min \{\beta, 1-\beta\}$ so that $\int l \lambda$ exists and is zero. For each $v \in F$ let

$$
g(v)=\left\{\begin{array}{lll}
1 & \text { if } \quad \beta(v)>\frac{1}{2} \\
0 & \text { if } \quad \beta(v) \leqq \frac{1}{2}
\end{array}=\min \{2(\beta(v)-l(v)), 1\}\right.
$$

Now by 2.K.2. $\int g \lambda$ exists and we have

$$
\begin{aligned}
\int g \lambda & =\int \min \{2(\beta-l), 1\} \lambda=\int \min \left\{2 \int \beta \lambda-2 \int l \lambda, \lambda\right\} \\
& =\int \min \left\{2 \int \beta \lambda, \lambda\right\}=\int \beta \lambda+\int \min \left\{\int \beta \lambda, \lambda-\int \beta \lambda\right\} \\
& =\int \beta \lambda-\int \min \{\beta, 1-\beta\} \lambda=\int \beta \lambda .
\end{aligned}
$$

If $D$ is a subdivision of $S$, i.e., a finite disjoint subset of $F$ whose union is $S$, then $H$ is a refinement of $D, H \ll D$, means that $H$ is a subdivision of $S$ and for each $v \in D$ there exists a subset $H_{v}$ of $H$ whose union is $v$.

Lemma 2. Suppose $\lambda \in b a(F)^{+},\left(E_{i}\right)$ is a disjoint sequence in $F, B>0$ and for each $i \in N$ we have $g_{i}: F \rightarrow[0, B]$ and $\int g_{i} \lambda$ exists. Suppose also that if $i \in N, I \in F$ and $g_{i}(I) \neq 0$, then $I \leqq E_{i}$. Then for each $v \in F, \int_{v} g(I) \lambda(I)$ exists and is $\sum_{i=1}^{\infty} \int_{v} g_{i}(I) \lambda(I)$, where $g(I)=$ $\sum_{i=1}^{\infty} g_{i}(I)$ for each $I \in F$.

Proof. Let $v \in F$ and $c>0$. Let $n$ be such that $\sum_{n}^{\infty} \lambda\left(E_{i} \cap v\right)<$ $c / 4 B$. For each $i \leqq n$ let $D_{i} \ll\left\{E_{i} \cap v\right\}$ be such that if $K \ll D_{i}$, then $\left|\sum_{K} g_{\imath}(I) \lambda(I)-\int_{\vartheta \cap E_{i}} g_{i}(I) \lambda(I)\right|<c / 2 n$. Let 


$$
D=\left(\bigcup_{i=1}^{n} D_{i}\right) \cup\left\{v \sim \bigcup_{i=1}^{n} E_{i}\right\}
$$

and suppose $H \ll D$. Let $H_{i}=\left\{I \in H \mid I \cong E_{i}\right\}$ for each $i$ and $H^{\prime}=$ $H \sim \bigcup_{i=1}^{n} H_{i}$. Note that if $I \in H_{i}$, then $g_{i}(I)=g(I)$. Now

$$
\begin{aligned}
&\left|\sum_{H} g(I) \lambda(I)-\sum_{1}^{\infty} \int_{v} g_{i}(I) \lambda(I)\right| \\
& \leqq\left|\sum_{i=2}^{n} \sum_{I I_{i}} g(I) \lambda(I)-\sum_{1}^{n} \int_{v \cap E_{i}} g_{i}(I) \lambda(I)\right| \\
&+\left|\sum_{H^{\prime}} g(I) \lambda(I)\right|+\left|\sum_{n+1}^{\infty} \int_{v \cap E_{i}} g_{i}(I) \lambda(I)\right| \\
& \leqq \sum_{\perp}^{n}\left|\sum_{H_{i}} g_{i}(I) \lambda(I)-\int_{v \cap E_{i}} g_{i}(I) \lambda(I)\right| \\
&+\sum\left\{g(I) \lambda(I) \mid I \in H^{\prime}, I \leqq E_{j} \cap v \quad \text { and } \quad j>n\right\} \\
&+\sum_{n+1}^{\infty} B \lambda\left(E_{i} \cap v\right) \\
&< \sum_{1}^{n} c / 2 n+\sum_{n+1}^{\infty} B \lambda\left(E_{j} \cap v\right)+B \cdot c / 4 B \\
& \leqq c / 2+B \cdot c / 4 B+c / 4=c .
\end{aligned}
$$

For $v \in F$ denote by $x_{v}$ the characteristic function of $\{I \in F \mid I \subseteq v\}$ and by $c_{v}(u)$ the contraction of $\mu$ to $v$, i.e., $c_{v}(\mu)=\int x_{v} \mu$.

A linear transformation, $T$, from $b a(F)$ into $b a(F)$ is in the class $\mathscr{C}$ [2] iff there exists a $K>0$ such that for each $v \in F$ and $\xi$ in $b a(F)$ we have

$$
\int_{v}|T(\xi)(I)| \leqq K \int_{v}|\xi(I)|
$$

THEOREM 3.A. If $T \in \mathscr{C}, \eta \in b a(F)^{+}$and $\delta \in A_{\eta}$, then $T(\delta)=$ $\int(T(\eta) / \eta) \delta$.

\section{Proof. [2].}

In [4] it was shown that the elements of $\mathscr{C}$ commute. Now, if $v \in F$ and $\lambda \in A_{\mu}^{+}$, then $c_{v}, \alpha_{\mu}$ and $\alpha_{\lambda}$ are clearly in $\mathscr{C}$. Therefore for $\xi \in b a(F)$ we have $\alpha_{\lambda}(\xi) \in A_{\mu}$, so that

$$
\text { 3.c.1. } \quad \alpha_{\lambda}(\xi)=\alpha_{\mu}\left(\alpha_{\lambda}(\xi)\right)=\alpha_{\lambda}\left(\alpha_{\mu}(\xi)\right)=\int\left(\alpha_{\lambda}(\mu) / \mu\right) \alpha_{\mu}(\xi),
$$

consequently if $\mu$ is representable, then $\lambda$ is also. If we replace $\lambda$, in 3.c.1, by $c_{v}(\mu)$ we have:

3.c.2.

$$
\alpha_{c_{v}(\mu)}(\xi)=\left(c_{v} \circ \alpha_{\mu}\right)(\xi),
$$


hence we may say that if $g$ represents $c_{v}(\mu)$ and $I \in F$ is such that $I \subseteq v$, then $g \cdot x_{I}$ represents $c_{I}(\mu)$.

4. The decomposition. Suppose $R \subseteq F$ is a ring of subsets of $S$ such that $I \in F$ and $I \subseteq v \in R$ implies that $I \in R$, then if $f$ is the characteristic function of $R$ and $\lambda \in b a(F)^{+}$the expression $\sum_{D} f(I) \lambda(I)$ is nondecreasing for successive refinements and bounded by $\lambda(S)$ so that $\int f \lambda$ exists.

THEOREM 1. Suppose $R \cong F$ is a ring of subsets of $S$ for which $I \in F$ and $I \subseteq v \in R$ imply $I \in R$. Suppose further that $c_{v}(\mu)$ is representable for each $v \in R$ and $\int f \mu=\mu$ where $f$ is the characteristic function of $R$. Then $\mu$ is representable.

Proof. Since $\mu=\int f \mu$ we have for each $n$ there exists $D_{n} \ll\{S\}$ such that if $E \ll D_{n}$, then $\mu(S)-\sum_{E} f(I) \mu(I)<1 / n$ and $D_{n}$ can be chosen so that $D_{n} \ll D_{n-1}$. Therefore if $v_{n}=\bigcup\left\{I \in D_{n} \mid f(I)=1\right\}$, then $v_{n} \subseteq v_{n+1}$ and $\mu\left(S \sim v_{n}\right)<1 / n$ for each $n$. Let $E_{1}=v_{1}$ and $E_{i}=v_{i} \sim$ $v_{i-1}$ for $i>1$. For each $i$ let $\mu_{i}=c_{E_{i}}(\mu)$ and $g_{i}: F \rightarrow\{0,1\}$ be such that $g_{i} \cdot x_{E_{i}}=g_{i}$ and $\alpha_{\mu_{i}}(\lambda)=\int g_{i} \lambda$ for each $\lambda \in b a(F)^{+}$. Let $g=\sum_{1}^{\infty} g_{i}$ and suppose $\lambda \in b a(F)^{+}$. By Lemma 2, $\int g \lambda$ exists and is $\sum_{1}^{\infty} \int g_{\imath} \lambda$ and for each $i$ we have $\alpha_{\mu_{i}}(\lambda)=\int g_{i} \lambda \in A_{\mu_{i}} \subseteq A_{\mu}$ and therefore $\int g \lambda \in A_{\mu}$. Thus, if $\lambda=\int g \lambda$, then $\lambda \in A_{\mu}$.

Now suppose $\lambda \in A_{\mu}^{+}$. Let $c>0$ and $n$ be such that $\mu(I)<1 / n$ implies that $\lambda(I)<c$. Then

$$
\begin{aligned}
0 & \leqq \lambda(S)-\int_{S} g(I) \lambda(I) \leqq \lambda(S)-\sum_{1}^{n} \int_{S} g_{i}(I) \lambda(I)=\lambda(S)-\sum_{1}^{n} \alpha_{\mu_{i}}(\lambda)(S) \\
& =\lambda(S)-\sum_{1}^{n} c_{E_{i}} \circ \alpha_{\mu}(\lambda)(S)=\lambda(S)-\sum_{1}^{n} \alpha_{\mu}(\lambda)\left(E_{i}\right) \\
& =\lambda(S)-\sum_{1}^{n} \lambda\left(E_{i}\right)=\lambda(S)-\lambda\left(v_{n}\right)=\lambda\left(S \sim v_{n}\right)<c .
\end{aligned}
$$

Therefore $\lambda \in A_{\mu}$ iff $\lambda=\int g \lambda$.

Now, as previously established, $\int g \lambda \in A_{\mu}$. Since $\int g \lambda \leqq \lambda$ it follows that $\int g \lambda \leqq \alpha_{\mu}(\lambda)=\int g \alpha_{\mu}(\lambda) \leqq \int g \lambda$, hence $g$ represents $\mu$.

If $\eta$ and $\delta$ are in $b a(F)^{+}$we will say that they are mutually singular if whenever $\lambda \in b a(F)^{+}$and $\lambda \leqq \eta$ and $\lambda \leqq \delta$, then $\lambda=0$. This is the notion of singularity used in [5] and [10] which is equivalent to that of [6]. It is also equivalent to $\int \min \{\eta, \delta\}=0$. 
Since $\eta$ and $\delta$ are only finitely additive we cannot, necessarily, obtain disjoint sets $s_{1}$ and $s_{2}$ such that $\eta\left(s_{1}\right)=\delta\left(s_{2}\right)=0$ with $s_{1} \cup s_{2}=t$.

THEOREM 2. There exist $\mu_{1}$ and $\mu_{2}$ in $b a(F)^{+}$such that:

(1) $\mu_{1}$ and $\mu_{2}$ are mutually singular and $\mu=\mu_{1}+\mu_{2}$.

(2) $\mu_{1}$ is representable.

(3) For each $v \in F$ we have $c_{v}\left(\mu_{2}\right)$ is representable iff $\mu_{2}(v)=0$.

(4) If $\mu_{3}$ is in $b a(F)^{+}, \mu_{2} \leqq \mu_{3} \leqq \mu$ and for each $v \in F$ we have $c_{v}\left(\mu_{3}\right)$ is representable iff $\mu_{3}(v)=0$, then $\mu_{2}=\mu_{3}$.

Proof. If $I, v \in F, I \subseteq v$ and $h$ represents $c_{v}(\mu)$, then by 3.c.2. $x_{I} \cdot h$ represents $c_{I}(\mu)$. Consequently $R=\left\{v \in F \mid c_{v}(\mu)\right.$ is representable $\}$ is a ring satisfying the conditions of Theorem 1 since for $I$ and $v$ in $R$ with $h, k$ representing $c_{I}(\mu)$ and $c_{v}(\mu)$ respectively we have $h+x_{v \sim I} \cdot k$ represents $c_{I \cup v}(\mu)$. Let $f$ be the characteristic function of $R$ and $\mu_{1}=\int f \mu$. Then for each $v \in R$ we have

$$
c_{v}\left(\mu_{1}\right)=\int x_{v} \mu_{1}=\int x_{v} \int f \mu=\int x_{v} f \mu=\int x_{v} \mu=c_{v}(\mu)
$$

so that $c_{v}\left(\mu_{1}\right)$ is representable. Also

$$
\int f \mu_{1}=\int f \int f \mu=\int f^{2} \mu=\int f \mu=\mu_{1}
$$

and thus, by Theorem $1, \mu_{1}$ is representable. Let $\mu_{2}=\mu-\mu_{1}=$ $\int(1-f) \mu$ and note that $\mu_{1}$ and $\mu_{2}$ are mutually singular since $\min \{f, 1-f\}=0$. Therefore for $\lambda \in b a(F)^{+}$we have $\alpha_{\mu_{1}}(\lambda)$ and $\alpha_{\mu_{2}}(\lambda)$ are mutually singular hence

$$
\alpha_{\mu_{1}}(\lambda)+\alpha_{\mu_{2}}(\lambda)=\int \max \left\{\alpha_{\mu_{1}}(\lambda), \alpha_{\mu_{2}}(\lambda)\right\} \leqq \alpha_{\mu}(\lambda) \leqq \alpha_{\mu_{1}}(\lambda)+\alpha_{\mu_{2}}(\lambda),
$$

i.e., $\alpha_{\mu_{1}}+\alpha_{\mu_{2}}=\alpha_{\mu}$. Now suppose $v \in F$ and $c_{v}\left(\mu_{2}\right)$ is representable, then $c_{v}(\mu)=c_{v}\left(\mu_{1}\right)+c_{v}\left(\mu_{2}\right)$ is representable so that $v \in R$. Therefore $f(I)=1$ for each $I \in F$ for which $I \subseteq v$. Hence

$$
\mu_{2}(v)=\int_{v}(1-f(I)) \mu(I)=0 .
$$

Finally suppose $\mu_{3} \in b a(F)^{+}$and $\mu_{2} \leqq \mu_{3} \leqq \mu$ and $c_{v}\left(\mu_{3}\right)$ is representable iff $\mu_{3}(v)=0$. For each $v \in R$ we have $c_{v}\left(\mu_{3}\right)$ is representable by 3.c.1. so that $\mu_{3}(v)=0$. Therefore

$$
\mu_{3}=\int f \mu_{3}+\int(1-f) \mu_{3} \leqq 0+\int(1-f) \mu=\mu_{2} .
$$

This decomposition differs from those of [6], [7] and [10] in that 
it does not give rise to a normal subspace [5]. To see that this is true suppose that the set $R$ of those elements of $b a(F)$ whose total variations are representable is a normal subspace and note that if $a \in b a(F)^{+}$and for each $v \in F$ we have $a(v) \in\{0, a(S)\}$, then $a \in R$. Therefore for any summable sequence, $\left(a_{n}\right)$, of such elements we have $\lambda=\sum_{n=1}^{\infty} \alpha_{n} \in R$. Consequently $\alpha_{\lambda}$ has an integral representation. However by [4] this is true iff the linear functional $\eta \rightarrow \alpha_{\lambda}(\eta)(S)$ has an integral representation and in [8] it was shown that this is not always true.

\section{REFERENCES}

1. W. D. L. Appling, Summability of real-valued set functions, Rivista Mathematica, University of Parma, VIII, No. 2 (1967), 77-100.

$2 . \quad$, Concerning a class of linear transformations, J. London Math. Soc., 44 (1969), 385-396.

3. - Addendum to: 'Some integral characterizations of absolute continuity,' Proc. Amer. Math. Soc., 24 (1970), 788-793.

4. — An isomorphism and isometry theorem for a class of linear functionals, Trans. Amer. Math. Soc., ICC (1974), 131-140.

5. S. Bochner and R. S. Phillips, Additive set functions and vector lattices, Ann. of Math., (2) 42 (1941), 131-140.

6. Richard B. Darst, A decomposition of finitely additive set functions, J. Reine Angew. Math., 210 (1962), 31-37.

7. E. Hewitt and K. Yoshida, Finitely additive measures, Trans. Amer. Math. Soc., 72 (1952), 46-66.

8. Michael Keisler, Integral representation for elements of the dual of $b a(c, \Sigma)$, preprint.

9. A. N. Kolmogoroff, Untersuchen über den Integralbegriff, Math. Annals, CIII (1930), 654-696.

10. Detlef Plachky, Decomposition of Additive Set Functions, Transactions of the Sixth Prague Conference on Information Theory, Satistical Decision Functions, Random Processes (Technical Univ. Prague, 1971; dedicated to the memory of Antonin Spacek), pp. 715-719. Academic, Prague, 1973.

Received October 29, 1976 and in revised form April 25, 1977.

Murray State University

MURRAY, KY 42071 



\title{
PACIFIC JOURNAL OF MATHEMATICS
}

\section{EDITORS}

\author{
RICHARD ARENS (Managing Editor) \\ University of California \\ Los Angeles, CA 90024 \\ Charles W. Curtis \\ University of Oregon \\ Eugene, OR 97403 \\ C. C. MOORE \\ University of California \\ Berkeley, CA 94720
}

\section{J. DUGUNDJI}

Department of Mathematics

University of Southern California

Los Angeles, CA 90007

R. FINN and J. MILGRAM

Stanford University

Stanford, CA 94305

\section{ASSOCIATE EDITORS}
E. F. BECKENBACH
B. H. NeumanN
F. WOLF
K. YoSHIDA

\section{SUPPORTING INSTITUTIONS}

UNIVERSITY OF BRITISH COLUMBIA

CALIFORNIA INSTITUTE OF TECHNOLOGY

UNIVERSITY OF CALIFORNIA

MONTANA STATE UNIVERSITY

UNIVERSITY OF NEVADA, RENO

NEW MEXICO STATE UNIVERSITY

OREGON STATE UNIVERSITY

UNIVERSITY OF OREGON

OSAKA UNIVERSITY

\author{
UNIVERSITY OF SOUTHERN CALIFORNIA \\ STANFORD UNIVERSITY \\ UNIVERSITY OF HAWAII \\ UNIVERSITY OF TOKYO \\ UNIVERSITY OF UTAH \\ WASHINGTON STATE UNIVERSITY \\ UNIVERSITY OF WASHINGTON \\ $\stackrel{*}{*} \stackrel{*}{*} \stackrel{*}{*}$ AMERICAN MATHEMATICAL SOCIETY
}

The Supporting Institutions listed above contribute to the cost of publication of this Journal, but they are not owners or publishers and have no responsibility for its content or policies.

Mathematical papers intended for publication in the Pacific Jaurnal of Mathematics should be in typed form or offset-reproduced, (not dittoed), double spaced with large margins. Please do not use built up fractions in the text of your manuscript. You may however, use them in the displayed equations. Underline Greek letters in red, German in green, and script in blue. The first paragraph or two must be capable of being used separately as a synopsis of the entire paper. Items of the bibliography should not be cited there unless absolutely necessary, in which case they must be identified by author and Journal, rather than by item number. Manuscripts, in triplicate, may be sent to any one of the editors. Please classify according to the scheme of Math. Reviews, Index to Vol. 39. All other communications should be addressed to the managing editor, or Elaine Barth, University of California, Los Angeles, California, 90024.

The Pacific Journal of Mathematics expects the author's institution to pay page charges, and reserves the right to delay publication for nonpayment of charges in case of financial emergency.

100 reprints are provided free for each article, only if page charges have been substantially paid. Additional copies may be obtained at cost in multiples of 50 .

The Pacific Journal of Mathematics is issued monthly as of January 1966. Regular subscription rate: $\$ 7200$ a year (6 Vols., 12 issues). Special rate: $\$ 36.00$ a year to individual members of supporting institutions.

Subscriptions, orders for back numbers, and changes of address should be sent to Pacific Journal of Mathematics, 103 Highland Boulevard, Berkeley, California, 94708.

PUBLISHED BY PACIFIC JOURNAL OF MATHEMATICS, A NON-PROFIT CORPORATION

Printed at Kokusai Bunken Insatsusha (International Academic Printing Co., Ltd.). 8-8, 3-chome, Takadanobaba, Shinjuku-ku, Tokyo 160, Japan.

Copyright (C) 1975 by Pacific Journal of Mathematics Manufactured and first issued in Japan 


\section{Pacific Journal of Mathematics}

Vol. 72, No. 2

February, 1977

George E. Andrews, Plane partitions. II. The equivalence of the

Bender-Knuth and MacMahon conjectures ................. 283

Lee Wilson Badger, An Ehrenfeucht game for the multivariable quantifiers

of Malitz and some applications ......................... 293

Wayne C. Bell, A decomposition of additive set functions ............ 305

Bruce Blackadar, Infinite tensor products of $C^{*}$-algebras ............. 313

Arne Brøndsted, The inner aperture of a convex set .............. 335

N. Burgoyne, Finite groups with Chevalley-type components........... 341

Richard Dowell Byrd, Justin Thomas Lloyd and Roberto A. Mena, On the retractability of some one-relator groups .....................

Paul Robert Chernoff, Schrödinger and Dirac operators with singular potentials and hyperbolic equations .................... 361

John J. F. Fournier, Sharpness in Young's inequality for convolution ....... 383

Stanley Phillip Franklin and Barbara V. Smith Thomas, On the metrizability

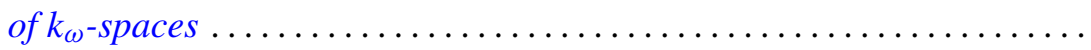

David Andrew Gay, Andrew McDaniel and William Yslas Vélez, Partially normal radical extensions of the rationals .................... 403

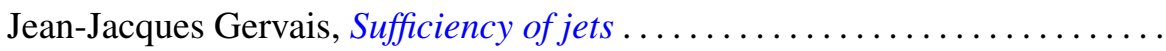

Kenneth R. Goodearl, Completions of regular rings. II . .

Sarah J. Gottlieb, Algebraic automorphisms of algebraic groups with stable maximal tori

Donald Gordon James, Invariant submodules of unimodular Hermitian forms.....

J. Kyle, $W_{\delta}(T)$ is convex.

Ernest A. Michael and Mary Ellen Rudin, A note on Eberlein compacts ...

Ernest A. Michael and Mary Ellen Rudin, Another note on Eberlein compacts ....

Thomas Bourque Muenzenberger and Raymond Earl Smithson, Fixed point theorems for acyclic and dendritic spaces.

Budh Singh Nashier and A. R. Rajwade, Determination of a unique solution of the quadratic partition for primes $p \equiv 1(\bmod 7)$.

Frederick J. Scott, New partial asymptotic stability results for nonlinear ordinary differential equations ....................

Frank Servedio, Affine open orbits, reductive isotropy groups, and dominant gradient morphisms; a theorem of Mikio Sato..........

D. Suryanarayana, On the distribution of some generalized square-full integers.................................. 